\title{
Negative Stiffness Demonstrated by NiAl Nanofilms
}

\author{
R.I. Babicheva ${ }^{1}$, K.A. Bukreeva ${ }^{2}$, S.V. Dmitriev ${ }^{2 *}$, R.R. Mulyukov ${ }^{2}$, K. Zhou ${ }^{1 *}$ \\ ${ }^{1}$ School of Mechanical and Aerospace Engineering, Nanyang Technological University \\ 50 Nanyang Avenue, Singapore 639798, Singapore \\ ${ }^{2}$ Institute for Metals Superplasticity Problems, Russian Academy of Sciences \\ 39 Khalturina St., Ufa 450001, Russia \\ *E-mail:dmitriev.sergey.v@gmail.com (S.V.Dmitriev),kzhou@ntu.edu.sg (K. Zhou)
}

Received: 9 February 2013; revised: 11 April 2013; accepted: 23 April 2013; published online: 28 May 2013

\begin{abstract}
This paper studies the uniaxial strain control tension of NiAl nanofilms via molecular dynamics simulations. The nanofilm deforms elastically until fracture at tensile strain is as large as $37 \%$. The stress-strain curve has a range where tensile deformation develops at decreasing tensile stress, thus indicating negative stiffness. Such deformation is thermodynamically unstable and the nanofilm splits into domains with two different values of elastic strain. Deformation within the unstable range is controlled by motion of the domain walls, resulting in the domains with larger strain grow at the expense of the domains with smaller strain.
\end{abstract}

Key words: negative stiffness; NiAl nanofilm; molecular dynamics; intermetallic compound

\section{INTRODUCTION}

Materials with negative Poisson's ratio [1-7], negative linear compressibility [7-13], negative thermal expansion [14-19], and negative refractive index [20,21] are wellknown examples of so-called negative materials. Such materials are interesting theoretically and from the practical point of view because their unusual properties can find new applications.

Negative materials are thermodynamically stable and their counterintuitive properties can be explained by peculiarities of their microstructures. On the other hand, free-standing negative-stiffness materials and structures are thermodynamically unstable; nevertheless, they are often observed experimentally and studied theoretically. The van der Waals equation of state for real gas and liquid describes isotherms that for sufficiently low temperatures possess a thermodynamically unstable range in the volume-pressure coordinates [22]. Within this range a mixture of gaseous and liquid phases is observed. A negative-shear modulus cell can be constructed by connecting pre-loaded elastic springs of different stiffnesses [23]. The von Mises frame, an arch or a spherical shell under lateral load exhibit the jump-like instability or, in the case of displacement control deformation, one can observe a decreasing part of the displacement-force curve [24]. Stiffness of the structures along this part of the curve is negative. Plastic deformation of metallic materials often shows the so-called yield drop resulting from either a dislocation unlocking mechanism or a dislocation multiplication at a very early stage of yielding [25].

For mechanical systems containing a negative-stiffness phase, anomalies in stiffness and damping have been observed experimentally [26,27] and described theoretically [28].

The concept of negative-stiffness-phase composites has been legitimized by Drugan by demonstrating for the two fundamental composite geometries the possibility of composite materials containing an isotropic phase having negative bulk and Young's moduli being stable overall, under merely applied traction boundary conditions, if the stable encapsulating phase is sufficiently stiff [29]. A thorough stability analysis for general composites when one phase violates positive-definiteness has been undertaken in the work [30].

Negative-stiffness elements can be used to improve vibration isolation systems [31,32]. Composite materials with 
negative-stiffness inclusions can have extreme overall stiffness and mechanical damping $[33,34]$. An elastic composite material having a component with sufficiently negative stiffness to produce positive-infinite composite stiffness can be stabilized by the gyroscopic forces produced by composite rotation [35].

It is well-known that nanomaterials very often have high strength approaching theoretical limit [36] and their deformation and fracture mechanisms can differ considerably from those operating in bulk materials [37]. In some cases nanowires under tension can show unusually large reversible strain. For instance, superelasticity in bcc nanowires by a reversible twinning mechanism has been observed in molecular dynamics simulations [38]. It has been shown that below a critical size composite trilayer nanowires deform via twinning and exhibit pseudoelastic behavior [39]. An ultra-high recoverable strain $(\sim 70 \%)$ has been revealed in hexagonal close-packed cobalt nanowires by molecular dynamics simulations, realized via lattice reorientation between $\langle\overline{1} 120\rangle$ and $\langle\overline{1} 100\rangle$ [40]. The pseudo-elasticity is found to be relevant to the cross-section size and length of the nanowires, which dominates the competition between structural transformation and stacking fault formation which leads to different degrees of pseudo-elasticity. Extremely large plastic deformation of intermetallic NiAl nanowire with failure strain as high as $700 \%$ at $700 \mathrm{~K}$ temperature has been reported [41]. Such a large elongation to failure is due to transformation from the crystalline phase to the amorphous phase after yielding of the nanowire.

In a series of works on atomistic simulation of $\mathrm{NiAl}$ and $\mathrm{CuZr}$ intermetallic nanowire tension, various interesting effects such as asymmetry under tensile/compressive loading and pseudo-elastic/pseudo-plastic behavior have been reported [42-45]. The structure transformations observed in the nanowires were called phase transitions. In the present study we demonstrate that no new phase appears during tension of $\mathrm{NiAl}$ nanofilms.

An interesting mechanism of two-phase stretching has been described for molecular chains by Savin et al. [46]. They have shown that, if the monomer stretching energy as a function of extension has a non-convex (concave up) region (see Fig. 1), the stretching of the polymer chain separates into two phases: weakly and strongly stretched monomers. Within the two-phase stretching regime, strain increases at constant stress. This is because the two-phase stretching does not follow the non-convex $P(\varepsilon)$ curve but develops along the lower energy path shown in Fig. 1 by the dashed line, which is the tangent to the $P(\varepsilon)$ curve. Linear $P(\varepsilon)$ dependence implies that $\sigma \sim d P / d \varepsilon$ is constant. For $\varepsilon<\varepsilon_{1}$ one has single-phase stretching. In the region $\varepsilon_{1}<\varepsilon<\varepsilon_{2}$ the domains with strain $\varepsilon_{1}$ and $\varepsilon_{2}$ coexist and elongation occurs due to the growth of the domains with larger strain in expense of the domains with smaller strain. When $\varepsilon$ reaches the value of $\varepsilon_{2}$, the domains with smaller strain disappear and further elongation develops homogeneously. Note that the use of the term "phase" in the work [46] is not actually justified because here one has not two different phases but the same phase at two different strain levels.

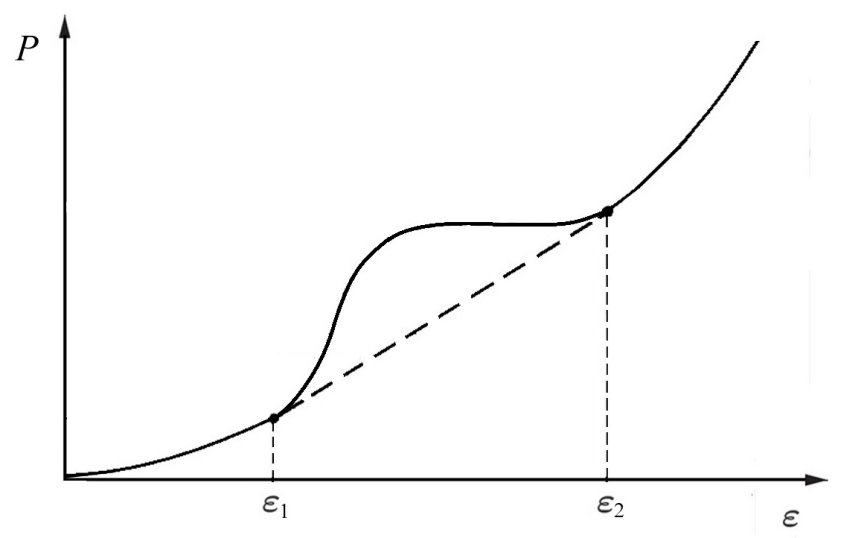

Fig. 1. Schema for the non-convex (concave up) dependence of the potential energy of a sample as the function of strain

It is also worth noting that Fig. 1 is used here for the purpose of illustration and, strictly speaking, it can be applied only to the analysis of $1 \mathrm{D}$ problems such as the problem discussed in [46]. In a general case one should speak about the loss of quasiconvexity of the $P(\varepsilon)$ curve.

Non-convex potentials similar to that shown in Fig. 1 have been employed to describe localization of plastic deformation and similar phenomena in frame of continuum mechanics $[47,48]$.

Surprisingly, in the present study, we find that a similar $P(\varepsilon)$ curve is demonstrated by the NiAl nanofilm under tension.

Many intermetallic compounds having bcc based B2 and fcc based $\mathrm{L}_{2}$ superstructures can show negative Poisson ratios for particular tension directions and directions of measuring lateral strain $[2,3]$, and thus they also belong to the class of negative materials.

Deformation mechanisms of pure metal nanowires and composite nanowires have been studied in a number of works. On the other hand, intermetallic nanomaterials have received very little attention. Intermetallic compounds have fewer slip systems than pure metals, which is why a larger elastic strain to failure can be expected for intermetallic nanomaterials.

This work, devoted to molecular dynamics simulations of uniaxial tension of NiAl nanofilm, is organized as follows. The simulation model is described in Sec. II, the numerical results are presented and discussed in Sec. III, and brief conclusions are given in Sec. IV.

\section{SIMULATION MODEL}

Uniaxial tension of an NiAl nanofilm is studied by molecular dynamic simulations using the LAMMPS program 
package. The interatomic potentials obtained by the embedded-atom method are employed [49]. The fitting database used in [49] for the development of the interatomic potentials includes experimental parameters (the lattice parameter $a_{0}$, the formation energy $E_{f}$, and the elastic constants $c_{i j}$ of the $\mathrm{B} 2$ structure of $\mathrm{NiAl}$ ) and the results of ab initio simulations (the nine formation energies of the $\mathrm{Ni}_{n} \mathrm{Al}_{m}$ compounds with different values of $\mathrm{n}$ and $\mathrm{m}$ having different ordered phases.

The Verlet method of order four with the time step of $1 \mathrm{fs}$ is used for numerical integration of the equations of motion of atoms.

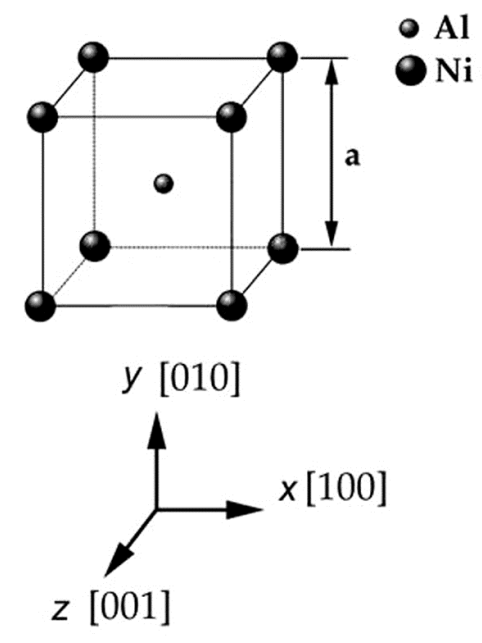

(a)

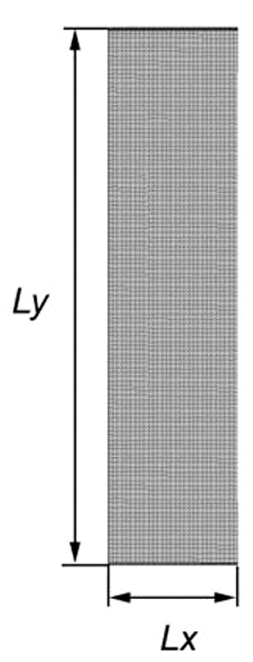

(b)
Fig. 2. (a) B2 superstructure based on the bcc lattice with the lattice parameter $a=2.8712 \AA$. $\mathrm{Al}(\mathrm{Ni})$ atoms are shown by small (large) spheres. (b) The computational cell having size $L_{x}=50 \mathrm{a}, L_{y}=200 \mathrm{a}$ and $L_{z}=3$ a subject to periodic boundary conditions along the $\mathrm{x}$ and $\mathrm{z}$ axes. Free surfaces of the nanofilm are parallel to the $\mathrm{xz}$ plane and normal to the $y$ axis. The nanofilm is subject to uniaxial tension along the $\mathrm{x}$ axis under the condition $\sigma_{y y}=\sigma_{z z}=0$

$\mathrm{NiAl}$ is an ordered intermetallic compound with B2 superstructure based on the bcc lattice with the lattice parameter $a=2.8712 \AA$ (see Fig. 2a). The Cartesian coordinate system is chosen so that the $\mathrm{x}$ and $\mathrm{z}$ axes are in the nanofilm plane and the $y$ axis is normal to the nanofilm plane (Fig.2b). The direction of tension of the nanofilm is along the $\mathrm{x}$ axis which coincides with[100] direction of bcc lattice. Free surfaces of the nanofilm are parallel to the (001) crystallographic planes.

A nanofilm of infinite size is modeled with the help of the periodic boundary conditions imposed along the $\mathrm{x}$ axis. The Parrinello-Rahman stress control is used to keep $\sigma_{z z}=$ 0 during tension along the $\mathrm{x}$ axis. Simulation results were compared for the computational cells of three different sizes, $L_{x}=3 a, L_{x}=50 a$, and $L_{x}=200 a$, with the same two other dimensions, $L_{y}=200 a$ and $L_{z}=3 a$. The three computational cells are referred to as small, intermediate, and large, respectively.

The film is subjected to strain control tension along the $\mathrm{x}$ axis with the strain rate of $0.5 \times 10^{9} \mathrm{~s}^{-1}$ for the small and intermediate computational cells and $1.25 \times 10^{8} \mathrm{~s}^{-1}$ for the large computational cell. It was checked numerically that such strain rates are sufficiently small to reproduce the domain wall dynamics in our case. Numerical simulations are conducted at temperature of $10 \mathrm{~K}$ controlled by the Nose-Hoover thermostat [50]. Small temperature is chosen to prevent early nucleation of dislocations at the nanofilm surfaces and by this means to extend the elastic range of deformation.

\section{NUMERICAL RESULTS AND DISCUSSIONS}

Firstly, the results for the small computational cell are presented. In Fig. 3a, we plot the tensile stress $\sigma_{x x}$ (solid line) and the potential energy per atom $P$ (dashed line) as the functions of tensile strain $\varepsilon_{x x}$. Note that $\sigma_{x x}$ is proportional to $d P / d \varepsilon_{x x}$. The curve $P\left(\varepsilon_{x x}\right)$ has a non-convex (concave up) shape between $\varepsilon_{x x}^{(1)}=0.158$ and $\varepsilon_{x x}^{(2)}=0.234$, which results in the appearance of the decreasing part on the $\sigma_{x x}\left(\varepsilon_{x x}\right)$ curve. For $\sigma_{x x}^{(1,2)} \leq \sigma_{x x} \leq \sigma_{x x}^{(3,4)}$, where $\sigma_{x x}^{(1,2)}=9.12 \mathrm{GPa}$ and $\sigma_{x x}^{(3,4)}=12.9 \mathrm{GPa}$, there are two possible values of strain corresponding to the 1-3 and 2-4 parts of the $\sigma_{x x}\left(\varepsilon_{x x}\right)$ curve. The decreasing part 3-2 corresponds to thermodynamically unstable states. In the stress control loading of the nanofilm, after reaching point 3 , strain would jump to point 4 at constant $\sigma_{x x}$. However, in the case of strain control loading employed in our simulations, the nanofilm deforms along the thermodynamically unstable path 3-2 demonstrating negative elastic modulus $E=d \sigma_{x x} / d \varepsilon_{x x}$, as can be seen in Fig. 3b. It should be kept in mind that the elastic modulus in the $\mathrm{y}$ and $\mathrm{z}$ directions is different from $\mathrm{E}$ because considerable homogeneous elastic strain makes the material of the nanofilm orthotropic. The elastic modulus as the function of tensile strain is plotted in Fig. $3 \mathrm{~b}$ by the thick line and the stress-strain curve is also reproduced here for reference by the thin line. Strain till global maximum with the coordinates $\varepsilon_{x x}=0.389, \sigma_{x x}=21.6 \mathrm{GPa}$ is elastic, which will be demonstrated later by comparing the loading and unloading curves for the computational cell of intermediate size.

Note that the $\sigma_{x x}\left(\varepsilon_{x x}\right)$ curve presented in Fig. 3a is qualitatively similar to the van der Waals real gas isotherm plotted in the volume-pressure coordinates [22]. It is well-known that in the thermodynamically unstable region the mixture of gaseous and liquid phases takes place. By analogy, for the considered nanofilm, from the stress-strain curve depicted in Fig. 3a one can expect coexistence of domains with larger and smaller elastic strain. The mixture of such domains would lower the total potential energy of the system. However, for such domains to appear the computational cell size should be sufficiently large. The size of the small computational cell used in our simulations is smaller than the threshold limit 

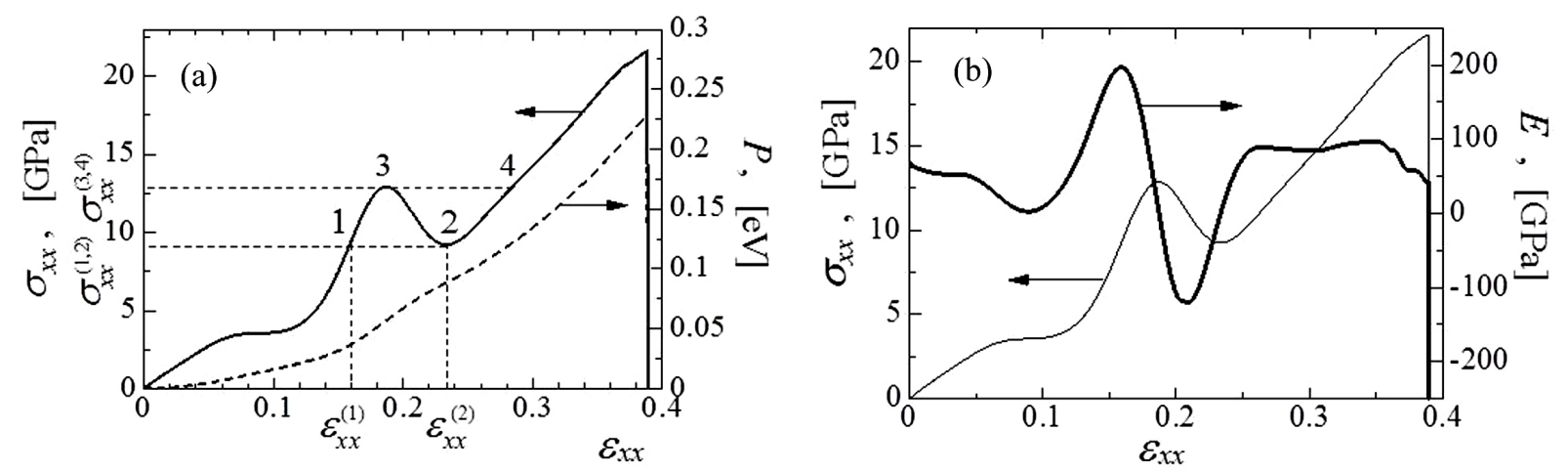

Fig. 3. Results for small computational cell: (a) tensile stress $\sigma_{x x}$ (solid line) and potential energy per atom $P$ (dashed line) as the functions of the uniaxial tensile strain $\varepsilon_{x x}$. For $\sigma_{x x}^{(1,2)} \leq \sigma_{x x} \leq \sigma_{x x}^{(3,4)}$ there are two possible values of strain on the lines 1-3 and 2-4. The line 3-2 corresponds to thermodynamically unstable states. Here $\varepsilon_{x x}^{(1)}=0.158, \varepsilon_{x x}^{(2)}=0.234$ and $\sigma_{x x}^{(1,2)}=9.12 \mathrm{GPa}, \sigma_{x x}^{(3,4)}=12.9 \mathrm{GPa}$. (b) Elastic modulus $E$ as the function of $\varepsilon_{x x}$ (thick line). The stress-strain curve is reproduced here by the thin line for reference. In the thermodynamically unstable region elastic modulus is negative

and the domains do not appear. In order to see the two-phase nanofilm stretching the simulations were repeated for the intermediate (shown in Fig. 2b) and large computational cells sizes.

Simulation results obtained for the intermediate computational cell are presented in Fig. 4a by dashed lines and for comparison the results obtained for the small simulation cell are reproduced here by the solid lines. Thin lines show $\sigma_{x x}\left(\varepsilon_{x x}\right)$, while thick lines present $E\left(\varepsilon_{x x}\right)$. The stress-strain curves for the intermediate computational cell also has a region with decreasing stress and hence, with negative elastic modulus. However, within the range of nonhomogeneous deformation of the nanofilm, $E$ is not the real material Young modulus but it is only a structural modulus.

In Fig. $4 \mathrm{~b}$ the loading and unloading curves in the thermodynamically unstable region are shown for the intermediate computational cell to demonstrate that the deformation is elastic. The loading and unloading curves coincide everywhere except for the regions where domains are nucleated. We also did not observe breaking or switching of interatomic bonds during deformation until fracture.

It can be seen from Fig. 4 that the results for the small and intermediate computational cells differ only within the unstable region and in the region close to the strength limit. The latter region is not interesting because here the difference in the behavior of the small and large computational cells can be easily explained by earlier fracture development in the larger cell. On the other hand, the former region is of special interest in the present study. The difference in the stress-strain curves obtained for the intermediate and small computational cells within the thermodynamically unstable region is due to the different deformation mechanisms. In the small computational cell, as it was stated earlier, the deformation develops homogeneously but in the intermediate computational cell in the thermodynamically unstable region the domains with smaller and larger elastic strain coexist. As a result, stretching occurs by an increase of the domains with larger strain at the expense of the domains with smaller strain.

In Fig. 4c,d we compare the stress-strain curves for the small, intermediate, and large computational cells, respectively. It can be seen that the results for the large cell differ from that for the intermediate cell only in the regions where nucleation of domains takes place. The negative slope in the regime of non-homogeneous stretching is the same for the intermediate and large computational cells.

Coming back to Fig. 4b we note that the difference between the loading and unloading curves in the thermodynamically unstable region is due to the nucleation of domains. Apart from the domain nucleation regions the loading and unloading stress-strain relations follow the same straight line with the negative slope. This negative slope indicates negative stiffness of the nanofilm and it can be explained by the existence of the Peierls-Nabarro barrier that should be overcome by the domain walls to move. One can expect that the negative slope would become smaller at higher temperatures because thermal fluctuations can help to overcome the Peierls-Nabarro barrier.

Under applied uniaxial load the primitive cell of the B2 superstructure is no longer cubic and has dimensions $a_{x}$, $a_{y}$ and $a_{z}$ along the $x, y$ and $z$ axes, respectively. Since $\sigma_{y y}=\sigma_{z z}=0$, one has $a_{y}=a_{z}$. Let us introduce local strain of the lattice cells as $e_{x}=\left(a_{x}-a\right) / a$ and $e_{y}=e_{z}=\left(a_{y}-a\right) / a$.

In Fig. 5, for the intermediate computational cell by blue (black) color we plot primitive cells with $e_{x}<0.2\left(e_{x}>0.2\right)$ for different values of the tensile strain $\varepsilon_{x x}$, which represent the average tensile strain of the nanofilm. In the thermodynamically stable regions the elastic strain of the nanofilm is homogeneous while in the unstable region the domains with larger and smaller $e_{x}$ can be seen. The domains are nucleated at the surfaces. In both types of domains the primitive cells have the shape of parallelepiped. Narrow domains then grow 

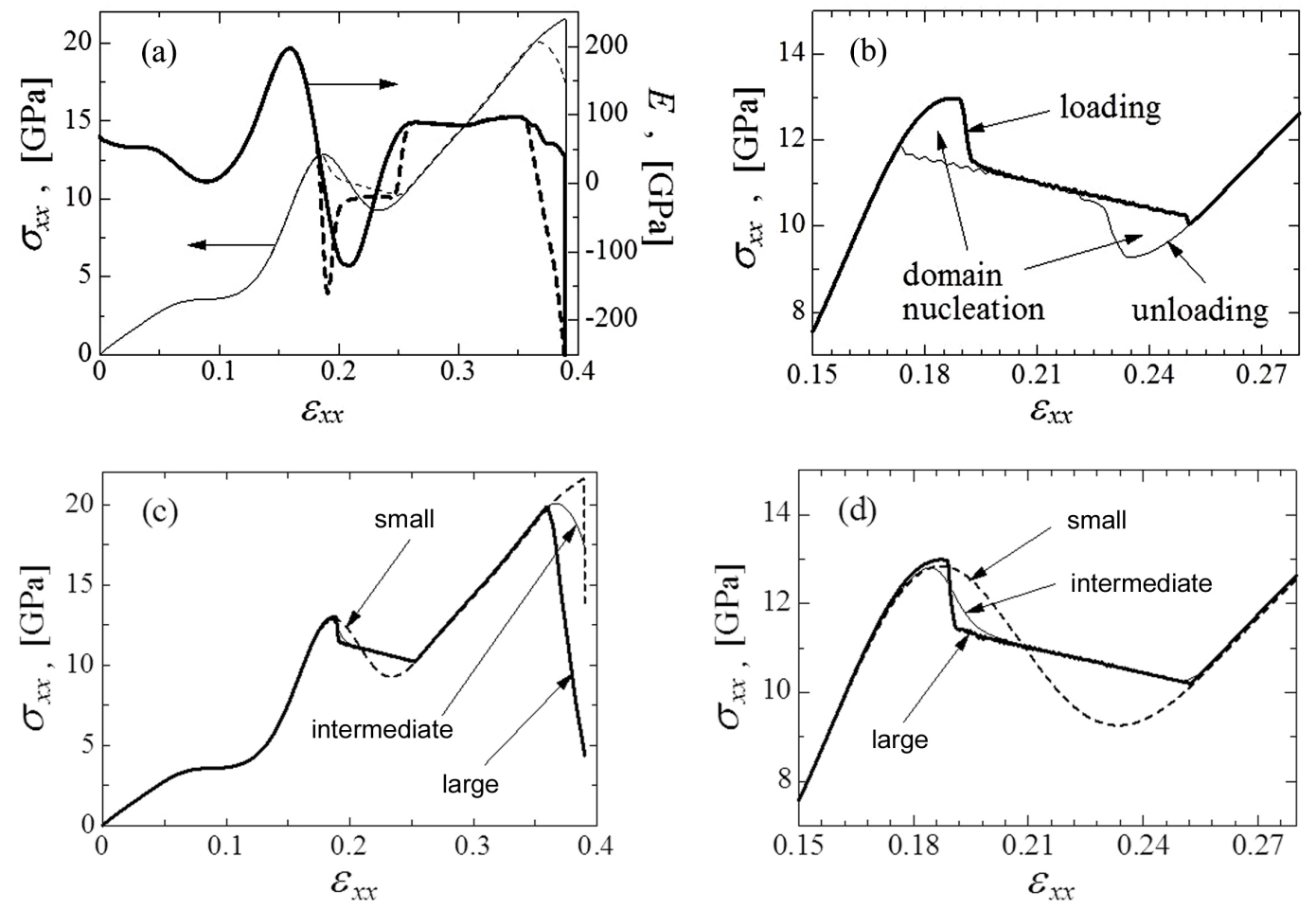

Fig. 4. (a) Results for small (solid lines) and large (dashed lines) computational cells. Thin lines show $\sigma_{x x}\left(\varepsilon_{x x}\right)$, while thick lines present $E\left(\varepsilon_{x x}\right)$. The results differ only in the thermodynamically unstable region and close to the fracture point. (b) Results for large computational cell. Loading and unloading curves in the thermodynamically unstable region. (c) Comparison of the stress-strain curves for the small (dashed line), intermediate (thin solid line), and large (thick solid line) computational cells. (d) Same as in (c) but in an enlarged scale

and merge into wider ones reducing total potential energy of the nanofilm by reducing total length of the domain walls that have relatively large energy. In the last panel of Fig. 5 one can see the crack nucleation that starts almost simultaneously in the bulk and at the surfaces.

In Fig. 6 we plot the same as in Fig. 5 but for the large computational cell. It can be seen that there is no qualitative difference in the domain nucleation and motion in the intermediate and large computational cells and this is the reason for nearly the same stress-strain curves in the thermodynamically unstable region obtained for the intermediate and large computational cells (see Fig. 4c,d).

In Fig. 7, we present local strain components $e_{x}$ and $e_{y}=e_{z}$ as the functions of the uniaxial tensile strain $\varepsilon_{x x}$. In the thermodynamically stable regions $e_{x}=\varepsilon_{x x}$. In the unstable region there exist two types of domains where $e_{x}=0.18$ and $e_{x}=0.25$ and these values do not change with an increase in $\varepsilon_{x x}$. The increase of $\varepsilon_{x x}$ in the unstable region occurs by growth of the domains with larger $e_{x}$ at the expense of the domains with smaller $e_{x}$. We stress that the two types of domains cannot be characterized as different phases because they have the same body-centered tetragonal symmetry. Thus, they are the domains of the same phase at two different strain levels.

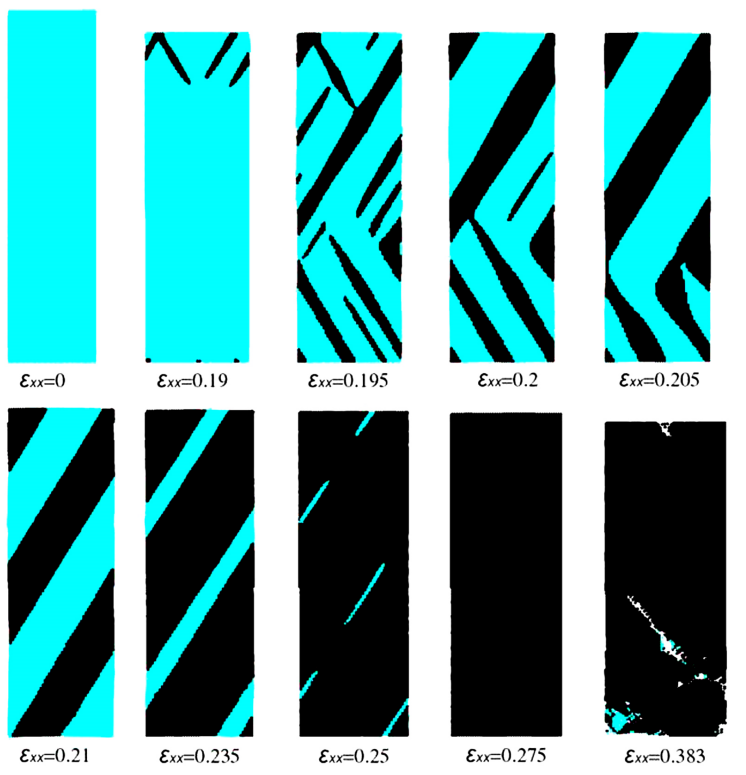

Fig. 5. Results for intermediate computational cell. Regions with lattice strain $e_{x}>0.2(<0.2)$ are shown in black (blue). At the domain walls separating blue and black areas the lattice strain sharply changes from $e_{x}=0.18$ to $e_{x}=0.25$. Average tensile strain $\varepsilon_{x x}$ is indicated for each panel 

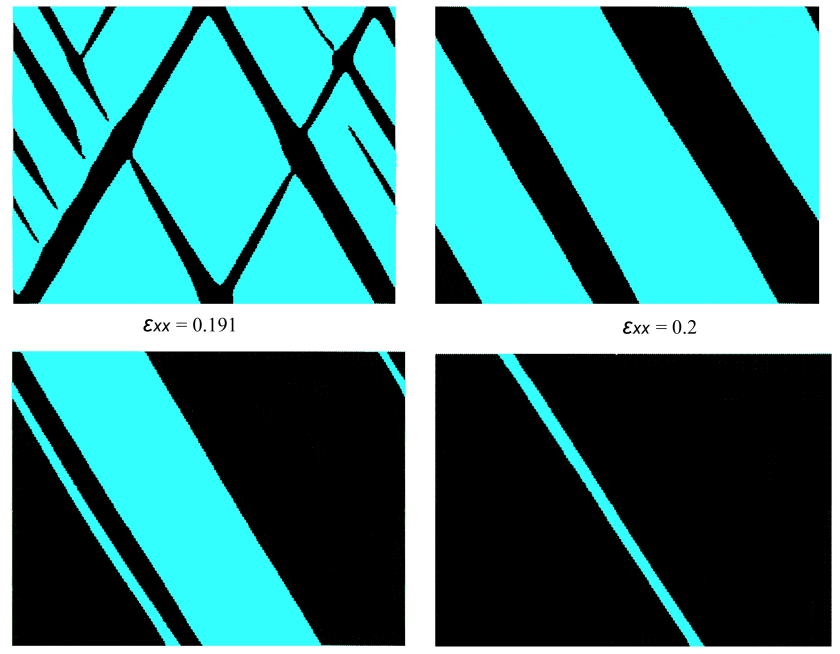

$\varepsilon x x=0.25$

Fig. 6. Same as in Fig. 5 but for the large computational cell

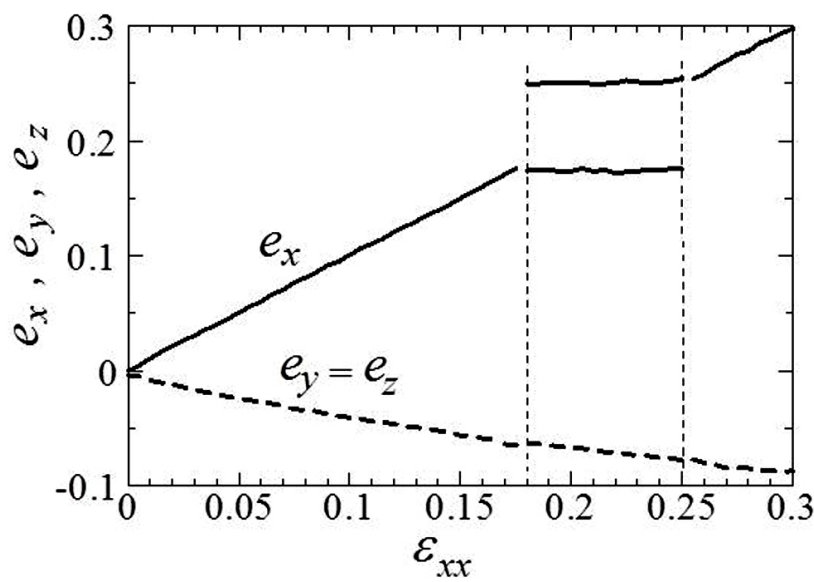

Fig. 7. Results for large computational cell: local lattice strains $e_{x}$ and $e_{y}=e_{z}$ as the functions of the uniaxial tensile strain $\varepsilon_{x x}$. In the thermodynamically stable regions $e_{x}=\varepsilon_{x x}$. In the unstable region there exist two types of domains where $e_{x}=0.18$ and $e_{x}=0.25$ and these values do not change with increase in $\varepsilon_{x x}$. Increase of $\varepsilon_{x x}$ in the unstable region occurs by growth of the domains with larger $e_{x}$ in expense of the domains with smaller $e_{x}$

A mechanism of two-phase deformation was recently reported by Savin et al. for the DNA molecule [46]. However, the DNA model is essentially one-dimensional, while in the case of nanofilm studied here the two-dimensional pattern of domains was observed. In fact, if in our simulations the computational cell in $z$ direction had a sufficiently large size (in our case $L_{z}=3 \mathrm{a}$ ), nucleation of the domains would become easier and the domain wall network could be even three-dimensional, if this is energetically preferable.

It is interesting to discuss why the curve $\sigma_{x x}\left(\varepsilon_{x x}\right)$ for the $\mathrm{NiAl}$ nanofilm shown in Fig. 3a has a decreasing region. We believe that this can hardly happen for pure metals, but for the ordered alloys and intermetallic compounds the appearance of thermodynamically unstable region could be a result of competing interaction between atoms of different sorts. The many-body interatomic potentials describing Ni-Ni, Al$\mathrm{Al}$ and $\mathrm{Ni}-\mathrm{Al}$ interactions have different strength and different effective length and their superposition results in the complex nonlinear elastic response observed for $\mathrm{NiAl}$ intermetallic compound.

\section{CONCLUSIONS}

Molecular dynamics simulation of NiAl nanofilm strain control uniaxial tension has revealed a region of tensile strain where elastic modulus obtains a negative value. Within this range the nanofilm is thermodynamically unstable. The small computational cell deforms homogeneously, but the sufficiently large computational cell in the unstable range splits into domains with larger and smaller strain. Deformation of the nanofilm in the unstable range develops by means of growth of the domains with larger strain at the expense of the domains with smaller strain. A similar two-phase stretching mechanism has been recently described for the DNA molecule [46]. An important difference of our work from the one-dimensional DNA case is that we observe a twodimensional domain pattern.

We believe that the appearance of the thermodynamically unstable region with negative slope on the $\sigma_{x x}\left(\varepsilon_{x x}\right)$ curve calculated for the NiAl nanofilm is a result of competing many-body interactions between atoms of different sorts. If this is so, it is unlikely that a pure metal would show negative elastic modulus.

In the future studies it is planned to investigate the effect of temperature on the nanofilm deformation through the thermodynamically unstable region. Boundary and loading conditions different from the strain-control loading will be analyzed in another publication. For the nonlinear physics community a detailed study of the domain wall dynamics would be interesting. The domain wall dynamics should be sensitive to the crystallographic orientations of the nanofilm with respect to the applied stress direction. It is also important to check if other intermetallic compounds can exhibit negative elastic modulus in a range of elastic strain.

\section{References}

[1] K. Evans, A. Alderson, Auxetic materials: Functional materials and structures from lateral thinking! Adv. Mater. 12, 617-628 (2000).

[2] A. Alderson, K.L. Alderson, Auxetic materials. Proceedings of the Institution of Mechanical Engineers, Part G: J Aerospace Eng. 221(4), 565-575 (2007).

[3] X.F. Wang, T.E. Jones, W. Li, Y.C. Zhou, Extreme Poisson's ratios and their electronic origin in $\mathrm{B} 2 \mathrm{CsCl}$-type $\mathrm{AB}$ intermetallic compounds. Phys. Rev. B 85, 134108-7 (2012). 
[4] T. Tian, X.F. Wang, W. Li, Ab initio calculations on elastic properties in L12 structure Al3X and X3Al-type (X=transition or main group metal) intermetallic compounds. Solid State Commun. 156, 69-75 (2013).

[5] J.W. Narojczyk, K.W. Wojciechowski, Elastic properties of degenerate f.c.c. crystal of polydisperse soft dimers at zero temperature. J. Non-Cryst. Solids 356, 2026-2032 (2010).

[6] A.A. Vasiliev, S.V. Dmitriev, Y. Ishibashi, T. Shigenari, Elastic properties of a two-dimensional model of crystals containing particles with rotational degrees of freedom. Phys. Rev. B 65, 094101-7 (2002).

[7] A.U. Ortiz, A. Boutin, A.H. Fuchs, F.-X. Coudert, Anisotropic elastic properties of flexible metal-organic frameworks: How soft are soft porous crystals? Phys. Rev. Lett. 109, 195502-5 (2012).

[8] A.D. Fortes, E. Suard, K.S. Knight, Negative linear compressibility and massive anisotropic thermal expansion in methanol monohydrate. Science 331, 742-746 (2011).

[9] J.N. Grima, D. Attard, R. Gatt, Unusual thermoelastic properties of methanol monohydrate. Science 331, 687-688 (2011).

[10] J.N. Grima, D. Attard, R. Caruana-Gauci, R. Gatt, Negative linear compressibility of hexagonal honeycombs and related systems. Scripta Mater. 65, 565-568 (2011).

[11] D.L. Barnes, W. Miller, K.E. Evans, A. Marmier, Modelling negative linear compressibility in tetragonal beam structures. Mech. Mater. 46, 123-128 (2012).

[12] E.V. Vakarin, A.V. Talyzin, On the mechanism of negative compressibility in layered compounds. Chem. Phys. 369, 1921 (2010).

[13] R.S. Lakes, K.W. Wojciechowski, Negative compressibility, negative Poisson's ratio, and stability. Phys. Status Solidi 245(3), 545-551 (2008).

[14] J.N. Grima, B. Ellul, D. Attard, R. Gatt, M. Attard, Composites with needle-like inclusions exhibiting negative thermal expansion: A preliminary investigation. Compos. Sci. Technol. 70, 2248-2252 (2010).

[15] V. Gava, A.L. Martinotto, C.A. Perottoni, First-principles mode Gruneisen parameters and negative thermal expansion in $\alpha-\mathrm{ZrW}_{2} \mathrm{O}_{8}$. Phys. Rev. Lett. 109, 195503-5 (2012).

[16] P.L. de Andres, F. Guinea, M.I. Katsnelson, Bending modes, anharmonic effects, and thermal expansion coefficient in single-layer and multilayer grapheme. Phys. Rev. B 86, 144103-5 (2012).

[17] V.E. Fairbank, A.L. Thompson, R.I. Cooper, A.L. Goodwin, Charge-ice dynamics in the negative thermal expansion material $\mathrm{Cd}(\mathrm{CN})_{2}$. Phys. Rev. B 86, 104113-5 (2012).

[18] A. Rebello, J.J. Neumeier, Z. Gao, Y. Qi, Y. Ma, Giant negative thermal expansion in La-doped $\mathrm{CaFe}_{2} \mathrm{As}_{2}$. Phys. Rev. B 86, 104303-6 (2012).

[19] I.A. Stepanov, Thermodynamics of substances with negative thermal expansion and negative compressibility. J. Non-Cryst. Solids 356, 1168-1172 (2010).

[20] V.G. Veselago, The electrodynamics of substances with simultaneously negative values of $\varepsilon$ and $\mu$. Soviet Physics Uspekhi. 10(4), 509-514 (1968).

[21] V. Veselago, L. Braginsky, V. Shklover, C. Hafner, Negative refractive index materials. J. Comput. Theor. Nanos. 3(2), 189-218 (2006).

[22] I.E. Dzyaloshinskii, E.M. Lifshitz, L.P. Pitaevskii, Reviews of topical problems: General theory of Van Der Waals' forces. Soviet Physics Uspekhi 4(2), 153-176 (1961).

[23] R.S. Lakes, W.J. Drugan, Dramatically stiffer elastic composite materials due to a negative stiffness phase? J. Mech. Phys. Solids. 50, 979-1009 (2002).
[24] D. Shilkrut, E. Riks, Stability of nonlinear shells: on the example of spherical shells. Elsevier Science, the Netherlands (2002), $458 \mathrm{p}$.

[25] X. Wang, H. Hamasaki, M. Yamamura, R. Yamauchi, T. Maeda, Y. Shirai, F. Yoshida, Yield-point phenomena of Ti20V-4Al-1Sn at $1073 \mathrm{~K}$ and its constitutive modelling. Mater. Trans. (JIM) 50(6), 1576-1578 (2009).

[26] T. Jaglinski, D. Kochmann, D. Stone, R.S. Lakes, Composite materials with viscoelastic stiffness greater than diamond. Science 315, 620 (2007).

[27] R.S. Lakes, T. Lee, A. Bersie, Y.C. Wang, Extreme damping in composite materials with negative-stiffness inclusions. Letters to Nature. Nature 410, 565-567 (2001).

[28] Y.C. Wang, J.G. Swadener, R.S. Lakes, Anomalies in stiffness and damping of a $2 D$ discrete viscoelastic system due to negative stiffness components. Thin Solid Films 515, 3171-3178 (2007).

[29] W.J. Drugan, Elastic composite materials having a negative stiffness phase can be stable. Phys. Rev. Lett. 98, 055502 (2007).

[30] D.M. Kochmann, W.J. Drugan, Analytical stability conditions for elastic composite materials with a non-positive-definite phase. Proc. R. Soc. A (2012) 468, 2230-2254.

[31] C.-M. Lee, V.N. Goverdovskiy, A multi-stage high-speed railroad vibration isolation system with "negative" stiffness. J. Sound Vib. 331, 914-921 (2012).

[32] A. Carrella, M.J. Brennan, T.P. Waters, K. Shin, On the design of a high-static-low-dynamic stiffness isolator using linear mechanical springs and magnets. J. Sound Vib. 315(3), 712-720 (2008).

[33] A.V. Dyskin, E. Pasternak, Elastic composite with negative stiffness inclusions in antiplane strain. Int. J. Eng. Sci. 58, 45-56 (2012).

[34] J. Yang, Y.P. Xiong, J.T. Xing, Dynamics and power flow behaviour of a nonlinear vibration isolation system with a negative stiffness mechanism. J. Sound Vib. 332(1), 167-183 (2013).

[35] D.M. Kochmann, W.J. Drugan, Infinitely stiff composite via a rotation-stabilized negative-stiffness phase. Appl. Phys. Lett. 99, 011909 (2011).

[36] T. Zhu, J. Li, Ultra-strength materials. Progr. Mater. Sci. 55(7), 710-757 (2010).

[37] J.R. Greer, J.T.M. De Hosson, Review: Plasticity in smallsized metallic systems: Intrinsic versus extrinsic size effect. Prog. Mater. Sci. 56(6), 654-724 (2011).

[38] S. Li, X. Ding, J. Deng, T. Lookman, J. Li, X. Ren, J. Sun, A. Saxena, Superelasticity in bcc nanowires by a reversible twinning mechanism. Phys. Rev. B 82, 205435-12 (2010).

[39] N. Abdolrahim, I.N. Mastorakos, H.M. Zbib, Deformation mechanisms and pseudoelastic behaviors in trilayer composite metal nanowires. Phys. Rev. B 81, 054117-5 (2010).

[40] C. Ni, H. Ding, C. Li, L.T. Kong, X.J. Jin, Pseudo-elasticity and ultra-high recoverable strain in cobalt nanowire: $A$ molecular dynamics study. Scripta Mater. 68, 191-194 (2013).

[41] V.K. Sutrakar, D.R, Mahapatra. Superplasticity in intermetallic NiAl nanowires via atomistic simulations. Mater. Lett. 64, 879-881 (2010).

[42] V.K. Sutrakar, D.R. Mahapatra, Asymmetry in structural and thermo-mechanical behavior of intermetallic NiAl nanowire under tensile/compressive loading: A molecular dynamics study. Intermetallics 18, 1565-1571 (2010).

[43] V.K. Sutrakar, D.R. Mahapatra, Stress-induced phase transformation and pseudo-elastic/pseudo-plastic recovery in intermetallic Ni-Al nanowires. Nanotechnology 20 , 295705-9 (2009). 
[44] V.K. Sutrakar, D.R. Mahapatra, Single and multi-step phase transformation in $\mathrm{CuZr}$ nanowire under compressiveltensile loading. Intermetallics 18, 679-687 (2010).

[45] V.K. Sutrakar, D.R. Mahapatra, Stress-induced martensitic phase transformation in $\mathrm{Cu}-\mathrm{Zr}$ nanowires. Mater. Lett. 63 , 1289-1292 (2009).

[46] A.V. Savin, I.P. Kikot, M.A. Mazo, A.V. Onufriev, Two-phase stretching of molecular chains. PNAS, doi:10.1073/pnas.1218677110 (2013).

[47] C. Miehe, M. Lambrecht, E. Gurses, Analysis of material instabilities in inelastic solids by incremental energy minimization and relaxation methods: evolving deformation microstructures in finite plasticity. J. Mech. Phys. Solids 52, 2725-2769 (2004).
[48] E.Gurses, C. Miehe, On evolving deformation microstructures in non-convex partially damaged solids. J. Mech. Phys. Solids 59, 1268-1290 (2011).

[49] G.P. Purja Pun,Y. Mishin, Development of an interatomic potential for the Ni-Al system. Phil. Mag. 89, 3245-3267 (2009).

[50] S. Nose, A unified formulation of the constant temperature molecular dynamics methods. J. Chem. Phys. 81, 511-519 (1984). 

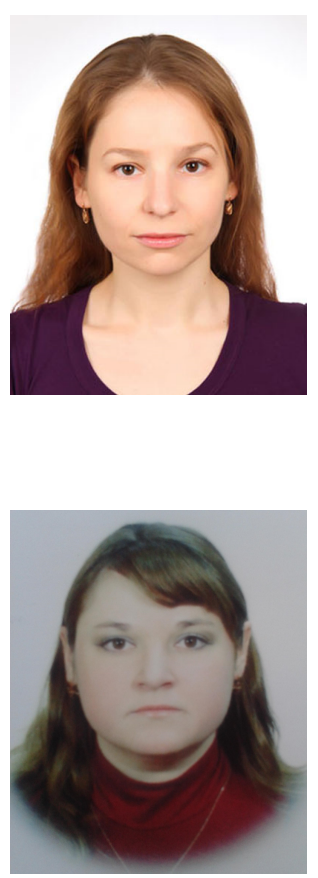

Rita I. BABICHEVA was born in Sterlitamak, Russia in 1985. She obtained her master's degree at the Ufa State Aviation Technical University, Russia in 2008. In the years 2008-2012, she worked as a trainee-researcher on the structure and functional properties of NiTi shape memory alloys in the Institute for Metals Superplasticity Problems, Russian Academy of Sciences, Ufa, Russia. Currently, she is a PhD student at the School of Mechanical and Aerospace Engineering, Nanyang Technological University, Singapore.

Karina A. BUKREEVA was born in Ufa, Russia in 1986. She graduated from the Ufa State Aviation Technical University in 2009. She is working at the Institute for Metals Superplasticity Problems, Russian Academy of Sciences, Ufa, Russia. Her scientific work in the field of Materials Science focuses on the mechanisms of elastic and plastic deformation of metallic and intermetallic nanomaterials studied with the help of computer simulations.

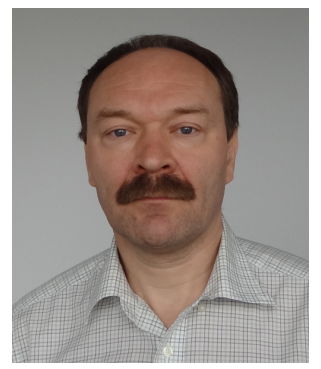

Sergey V. DMITRIEV was born in 1961 in Tomsk, Russia. S.V. Dmitriev graduated from Tomsk State University with an M.S. degree in 1984. Completed the $\mathrm{PhD}$ degree in structural mechanics at Tver State University, Russia, in 1988. Gained the second $\mathrm{PhD}$ degree in computer modeling of incommensurate phases in dielectrics from the University of Electro-Communications, Tokyo, Japan, in 1999. Received a Habilitation in 2008 with the thesis on solitary wave dynamics in discrete systems from the Altai State Technical University, Barnaul, Russia. Since 2008, S.V. Dmitriev has been serving as the Head of Laboratory of the Institute for Metals Superplasticity Problems, Ufa, Russia. His scientific interests are in solitary waves in discrete systems; discrete breathers; evolution of defect structures in solids during plastic deformation; mechanical properties of graphene; atomistic simulations.

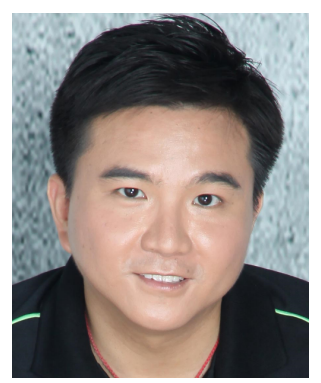

Kun ZHOU was born in Hunan Province, China. He obtained his $\mathrm{PhD}$ in mechanical engineering from Nanyang Technological University (NTU), Singapore in 2006, and his M. E. and B. E. in mechanical engineering from Tsinghua University, China in 2001 and 1998, respectively. From 2007 to 2010, he worked as a Postdoctoral Fellow at the Center for Surface Engineering \& Tribology, Northwestern University, USA. Since 2010, he has been an Assistant Professor at the School of Mechanical and Aerospace Engineering, NTU. His research interests focus on micro/nano mechanics of materials and structures, novel computational methods for modeling material behavior, and sustainable energy and green technologies. He currently serves on the Elasticity Technical Committee, American Society of Civil Engineers (ASCE). 